\title{
Association of Clinical and Laboratory Variables with In-Hospital Incidence of Deep Vein Thrombosis in Patients with Acute Stroke: A Retrospective Study
}

\section{Yucai Huang}

Changsha central hospital

\section{Kun Song}

Changsha Central Hospital

\section{Wen Peng}

Second Xiangya Hospital

Yang Zhou

Second Xiangya Hospital

\section{Qiong Ding}

Changsha Central Hospital

\section{Changluo Li}

Changsha Central Hospital

Ning Ding ( $\nabla$ doctordingning@csu.edu.cn )

Changsha Central Hospital

\section{Original Research}

Keywords: deep vein thrombosis, stroke, pulmonary infection, muscle tone, D-dimer

Posted Date: July 6th, 2020

DOl: https://doi.org/10.21203/rs.3.rs-39406/v1

License: (c) (i) This work is licensed under a Creative Commons Attribution 4.0 International License. Read Full License 


\section{Abstract}

Backgrounds: Deep vein thrombosis (DVT) is a serious complication in patients with acute stroke. Early prediction of DVT could enable physicians to perform a proper prevention strategy. We analyzed the association of clinical and laboratory variables with DVT to evaluate the risk of DVT in patients after stroke.

Results: Acute stroke patients admitted to the Changsha Central Hospital between January 2016 and December 2019 with length of stay in hospital $\geq 30 \mathrm{~d}$ were included. Clinical and laboratory variables for DVT at baseline were collected, and the diagnosis of DVT was confirmed by ultrasonography. Risk factors were developed by Multivariate logistic regression analysis. A total of 101 patients were included in the study, of which $77.2 \%(78 / 101)$ were diagnosed ischemic stroke and $22.8 \%(23 / 101)$ were confirmed hemorrhagic stroke. The overall incidence of DVT after acute stroke within 30 days was $19.8 \%(20 / 101)$. The level of D-dimer when DVT detected was significant increased than that on admission. Pulmonary infection and increased muscle tone as independent relevant factors for DVT were confirmed.

Conclusions: Pulmonary infection as a risk factor and increased muscle tone as a protector factor for DVT were identified in patients after stroke and the significant change in level of D-dimer could be a warning for DVT occurrence. It helps with taking early managements to reduce the incidence of DVT in patients after stroke.

\section{Introduction}

Stroke is one of the severe cerebral disorders leading to high disability and mortality among hospitalized patients(1), while deep vein thrombosis (DVT) is one of most common and fatal complications in patients after stroke(2). In western countries, the incidence of DVT in acute stroke patients without prophylactic treatment was up to around $80 \%(3)$, and DVT still occurred in at $2-3 \%$ of patients even receiving comprehensive prophylactic therapy $(4,5)$. The CLOTS trial, as a largest multicenter observational research with 5632 patients with stroke revealed that the in-hospital incidences of detected DVT within 10 days and within 30 days were $11 \%$ and 15\%, respectively(6). In Asia, the morbidity of DVT after stoke was less common, which varied in $3-17 \%(7)$.

However, the clinical outcomes were confirmed to be improved significantly in DVT patients after stroke including thrombolytic and anti-coagulation therapies(8), there was still lack of explicitness on the timing of thromboprophylaxis in the international guidelines(1). Hence, the risk of developing DVT in every single patient after stroke should be evaluated early so that the benefit and risk of thromboprophylaxis therapy could be comprehensively weighed and analyzed. In this study, we analyzed the association of clinical and laboratory variables with DVT in patients after stroke in order to explore risky factors for predicting inhospital incidence of DVT.

\section{Methods}




\section{Patients}

Acute stroke patients admitted to the Changsha Central Hospital between January 2016 and December 2019 with length of stay(LOS) in hospital $\geq 30 \mathrm{~d}$ were included. Inclusion criteria were identified as follows: age $\geq 18$, radiographical results showing cerebral infarction or cerebral hemorrhage, and length of stay in-hospital $\geq 30 \mathrm{~d}$. Exclusion criteria were identified as follows: past medical history of DVT, DVT detected on admission, varicose of lower extremities, malignant tumor and coagulation disorder.

\section{Data collection}

Based on electronic health records, the general information of patients was collected, including age ,sex and comorbidities (coronary heart disease, rheumatoid heart disease, atrial fibrillation, hypertension, diabetes). The National Institutes of Health Stroke Scale(NIHSS), Glasgow Coma Scale(GCS), Wells scale and Modified Rankin Scale(MRS) were performed for all the patients when on admission. Laboratory variables while patients admitted in $\leq 24 \mathrm{~h}$ were collected including platelet counts, red blood cell volume distribution width(RDW), low density lipoprotein(LDL), D-dimer and fibrinogen (Fbg). Moreover, management therapies including anti-coagulation and rehabilitation were also recorded. When DVT detected in patients by color doppler ultrasonography (CDUS), the clinical variables in 24 hours were collected. The incidence of in-hospital pulmonary infection and in-bed $\geq 3 \mathrm{~d}$ were recorded. Clinical outcomes were LOS in hospital, in-hospital incidence of pulmonary embolism and in-hospital mortality.

\section{DVT assessment}

According to the electronic health record, the DVT assessment was applied with color doppler ultrasonography (CDUS) on the patients while on admission. Common femoral vein and the popliteal vein of patients were examined by CDUS for DVT diagnosis. During the time of in-hospital, CDUS was performed in 2 weeks after admission as well as whenever clinically requested such as swollen or paresthesia of extremities on the basis of electronic health records.

\section{Statistics}

Statistical results were showed in mean \pm standard deviation for normal data, while for non-normal data, interquartile range (IQR)and median were utilized. Categorical data were showed as percentage and number. The comparison between two groups was performed with chi-squared test or Mann-Whitney Utest. Variables that were significant different in two groups on univariate analysis were further analyzed in multivariate logistic regression. Statistical analysis was performed using SPSS software (version 26) and two-sided $P$ values of less than 0.05 were defined statistically significant.

\section{Results}




\section{General characteristics of the patients}

A total of 122 patients with diagnosis of acute stroke were enrolled and 21 were excluded on the basis of exclusion criteria(Fig. 1). Finally, 101 patients were included in the study, of which $77.2 \%(78 / 101)$ were diagnosed ischemic stroke ,22.8\%(23/101)were confirmed hemorrhagic stroke, 66\% (67/101)were male and median age was $66(66.0 \pm 16.2)$. There were 20 patients in DVT group and 81 patients in non-DVT group, respectively. The general characteristics of the patients were demonstrated in Table 1. There were no significant difference in proportion of subtypes of stroke, sex, age, comorbidities (coronary heart disease, rheumatoid heart disease, atrial fibrillation, hypertension, diabetes) between two groups. Lab variables(platelet counts, RDW, LDL, fibrinogen(Fbg), management(anti-coagulation, rehabilitation therapy) and clinical outcomes were no significant different between two groups. None of the patients had in-hospital pulmonary embolism. In DVP group, the proportion of increased muscle tone was significant lower than that in non-DVT group( $10 \%$ vs $67.8, P=0.002)$, while there was no significant difference in muscle strength between two groups. The incidence of pulmonary infection was significant higher in DVT group than non-DVT group( $85 \%$ vs $60.4 \%, P=0.044)$. The level of $D$-dimer and Wells scale were also significant different $(P<0.05)$. 
Table 1

Baseline characteristics of the patients $(n=101)$

\begin{tabular}{|c|c|c|c|c|}
\hline Characteristic & Total $(n=101)$ & DVT $(n=20)$ & Non-DVT $(n=81)$ & P-value \\
\hline Gender & $67(66.3)$ & $11(55.0)$ & $56(69.1)$ & 0.292 \\
\hline \multicolumn{5}{|l|}{ Male, $\mathrm{n}(\%)$} \\
\hline Female, n(\%) & $34(33.7)$ & $9(45.0)$ & $20(30.9)$ & \\
\hline Age (years, mean \pm sd) & $66.0 \pm 16.2$ & $65 \pm 16.4$ & $66 \pm 16.3$ & 0.684 \\
\hline Ischemic stroke, n(\%) & 78(77.2) & $15(75.0)$ & $63(77.8)$ & 0.938 \\
\hline Hemorrhagic stroke & $23(22.8)$ & $4(20.0)$ & 19(23.4) & 0.626 \\
\hline \multicolumn{5}{|l|}{$\mathrm{n}(\%)$} \\
\hline \multicolumn{5}{|l|}{ Comorbidities } \\
\hline Coronary heart disease, $\mathrm{n}(\%)$ & $23(22.7)$ & $8(40.0)$ & 15(18.5) & 0.070 \\
\hline Rheumatoid heart disease, $\mathrm{n}(\%)$ & $6(5.9)$ & $1(5.0)$ & $5(6.1)$ & 1.000 \\
\hline Arial fibrillation, n(\%) & 19(18.8) & $7(35.0)$ & $12(21.4)$ & 0.055 \\
\hline Hypertension, n(\%) & $83(82.1)$ & $18(90.0)$ & $65(80.2)$ & 0.514 \\
\hline Diabetes ,n(\%) & $15(14.8)$ & $2(10.0)$ & $13(16.0)$ & 0.729 \\
\hline \multicolumn{5}{|l|}{ In-hospital complications } \\
\hline In-bed $\geq 3 \mathrm{~d}, \mathrm{n}(\%)$ & $85(84.1)$ & $17(85.0)$ & 68(83.9) & 1.000 \\
\hline Pulmonary infection, $\mathrm{n}(\%)$ & $66(65.3)$ & $17(85.0)$ & $49(60.4)$ & 0.044 \\
\hline Central venous catheter, n(\%) & $6(5.9)$ & $2(10.0)$ & $4(4.9)$ & 0.340 \\
\hline Increased muscle tone n(\%) & $40(39.6)$ & $2(10.0)$ & $38(67.8)$ & 0.002 \\
\hline Muscle strength (grade, mean $\pm s d$ ) & $2.4 \pm 1.6$ & $2.8 \pm 1.7$ & $2.4 \pm 1.6$ & 0.332 \\
\hline \multicolumn{5}{|l|}{ Lab findings } \\
\hline Platelet $\left(10^{\wedge} 9 / \mathrm{L}\right.$, mean $\left.\pm \mathrm{sd}\right)$ & $189.3 \pm 57.7$ & $182 \pm 50.5$ & $191 \pm 59.5$ & 0.512 \\
\hline RDW(\%,mean \pm sd $)$ & $14.6 \pm 12.3$ & $13.7 \pm 2.1$ & $14.9 \pm 1.5$ & 0.704 \\
\hline $\mathrm{LDL}(\mathrm{mmol} / \mathrm{L}$, mean $\pm \mathrm{sd}))$ & $2.0 \pm 1.2$ & $1.9 \pm 1.2$ & $2.0 \pm 1.1$ & 0.813 \\
\hline $\mathrm{D}$-dimer$(\mathrm{mg} / \mathrm{L}$, mean $\pm \mathrm{sd})$ & $1.6 \pm 3.3$ & $3.1 \pm 5.3$ & $1.3 \pm 2.4$ & 0.021 \\
\hline $\mathrm{Fbg}(\mathrm{mg} / \mathrm{L}$, mean $\pm \mathrm{sd})$ & $2.9 \pm 1.3$ & $2.5 \pm 1.1$ & $3.0 \pm 1.3$ & 0.068 \\
\hline \multicolumn{5}{|c|}{$\begin{array}{l}\text { Abbreviations: DVT = Deep Vein Thrombosis, RDW = Red blood cell volume distribution width, LDL = } \\
\text { low density lipoprotein, Fbg = fibrinogen,GCS = Glascow Coma ScalenNIHSS = National Institutes of } \\
\text { Health Stroke Scale, MRS = Modified Rankin Scale }\end{array}$} \\
\hline
\end{tabular}




\begin{tabular}{|c|c|c|c|c|}
\hline Characteristic & Total $(n=101)$ & DVT $(n=20)$ & Non-DVT $(n=81)$ & P-value \\
\hline \multicolumn{5}{|l|}{ Scoring system } \\
\hline GCS & $11.6 \pm 3.5$ & $11.5 \pm 3.5$ & $11.6 \pm 3.5$ & 0.927 \\
\hline NIHSS & $16.9 \pm 10.2$ & $19.7 \pm 10.6$ & $16.2 \pm 10.1$ & 0.181 \\
\hline MRS & $4.1 \pm 1.3$ & $4.2 \pm 1.4$ & $4.1 \pm 1.3$ & 0.905 \\
\hline Wells & $0.43 \pm 0.64$ & $0.7 \pm 0.7$ & $0.4 \pm 0.6$ & 0.031 \\
\hline \multicolumn{5}{|l|}{ Management } \\
\hline Anti-coagulation $\mathrm{n}(\%)$ & $8(7.9)$ & $1(5.0)$ & $7(8.6)$ & 1.000 \\
\hline Rehabilitation therapy n(\%) & $101(100.0)$ & $20(100.0)$ & $81(100.0)$ & 1.000 \\
\hline \multicolumn{5}{|l|}{ Clinical outcomes } \\
\hline Pulmonary embolism n(\%) & $0(0.0)$ & $0(0.0)$ & $0(0.0)$ & 1.000 \\
\hline Length of stay in hospital(days) & $79.0 \pm 60.2$ & $73.7 \pm 37.6$ & $80.3 \pm 64.7$ & 0.666 \\
\hline In-hospital mortality, n(\%) & $1(0.9)$ & $0(0.0)$ & $1(1.2)$ & 1.000 \\
\hline \multicolumn{5}{|c|}{$\begin{array}{l}\text { Abbreviations: DVT = Deep Vein Thrombosis, RDW = Red blood cell volume distribution width, LDL = } \\
\text { low density lipoprotein, Fbg = fibrinogen,GCS = Glascow Coma Scale } 1 \text { NIHSS = National Institutes of } \\
\text { Health Stroke Scale, MRS = Modified Rankin Scale }\end{array}$} \\
\hline
\end{tabular}

\section{Multiple logistic regression analysis for in-hospital incidence of DVT}

Two independent variables were identified by multivariate logistic regression analysis in (Table 2).

Pulmonary infection was a risk factor for in-hospital incidence of DVT( Odds Ratio(OR) $=5.4,95 \% \mathrm{Cl}: 1.10$ $26.65, P=0.037)$ ), while increased muscle tone was negative parallel with in-hospital incidence of $\operatorname{DVT}(\mathrm{OR}=0.11,95 \% \mathrm{Cl}=0.02-0.58, \mathrm{P}=0.010)$. 
Table 2

Multiple logistic regression analysis for in-hospital incidence of

DVT

\begin{tabular}{|lcll|}
\hline & OR & $95 \% \mathrm{Cl}$ & p-value \\
\hline Coronary heart disease & 2.4 & $0.48-12.24$ & 0.288 \\
\hline Atrial fibrillation & 2.5 & $0.49-13.2$ & 0.265 \\
\hline Fbg & 0.6 & $0.34-1.13$ & 0.116 \\
\hline D-dimer & 1.2 & $0.91-1.47$ & 0.231 \\
\hline Increased muscle tone & 0.11 & $0.02-0.58$ & 0.010 \\
\hline Wells score & 2.0 & $0.86-4.62$ & 0.106 \\
\hline Pulmonary infection & 5.4 & $1.10-26.65$ & 0.037 \\
\hline Abbreviations: DVT = Deep Vein Thrombosis, Fbg = fibrinogen \\
\hline
\end{tabular}

\section{Analysis of the relevance between DVT and increased muscle tone}

In non-DVT group, the incidence of increased muscle tone was significant higher than that in DVT group(67.8\%vs10\%,P = 0.002)(Fig. 2). Non-parametric correlation analysis showed that there was a negative correlation between DVT and increased muscle tone $(R=-0.703, P=0.031)$. Compared the time when DVT detected and when muscle tone increased, the average days from admission to when DVT detected were significantly longer than the time from admission to when muscle tone increased(17.7 vs $9.9, P<0.001)$ (Fig. 3).

\section{Analysis of the changes in laboratory variables when DVT detected}

Compared the levels of laboratory variables in patients with DVT between the time on admission and the time when DVT detected, the level of D-dimer when DVT detected was significant increased than that on admission $(P<0.001)$ (Table 3$)$. 
Table 3

Comparison laboratory variables in different time

\begin{tabular}{|llll|}
\hline & At admission & At Time when DVT detected & p-value \\
\hline Platelet $\left(10^{\wedge} 9 / \mathrm{L}\right.$, mean $\left.\pm \mathrm{sd}\right)$ & $182 \pm 50.5$ & $172 \pm 30.5$ & 0.483 \\
\hline $\mathrm{RDW}(\%$, mean $\pm \mathrm{sd})$ & $13.7 \pm 2.1$ & $13.9 \pm 4.9$ & 0.658 \\
\hline $\mathrm{LDL}(\mathrm{mmol} / \mathrm{L}$, mean $\pm \mathrm{sd}))$ & $1.9 \pm 1.2$ & $1.8 \pm 1.3$ & 0.337 \\
\hline $\mathrm{D}-\mathrm{dimer}(\mathrm{mg} / \mathrm{L}$, mean $\pm \mathrm{sd})$ & $3.1 \pm 5.3$ & $13.6 \pm 1.7$ & $<0.001$ \\
\hline Fbg(mg/L, mean $\pm \mathrm{sd})$ & $2.5 \pm 1.1$ & $2.8 \pm 0.9$ & 0.198 \\
\hline Abbreviations: $\mathrm{DVT}=$ Deep Vein Thrombosis, RDW = Red blood cell volume distribution width \\
\hline
\end{tabular}

\section{Discussion}

Risk factors for DVT in patients after acute stroke varied in different clinical researches. The typical factors included older age, medical history of DVT, increased body mass index(BMI), malignant tumor, pulmonary infection, increased level of some laboratory variables(9-13). In our study, pulmonary infection and increased muscle tone were identified as independent factors associated with in-hospital incidence of DVT in patients after acute stroke.

In our multiple logistic regression model, patients with pulmonary infection experienced an increased risk of DVT. A higher risky relevance of pulmonary infection with DVT was also demonstrated in other researches $(14,15)$. A research on the psychiatric inpatients revealed that the average in-hospital incidence of DVT was up to $10 \%$, while the DVT risk in the group with pulmonary infection was significantly increased(16). In addition, a clinical case review showed that patients died secondary to staphylococcal community-acquired pneumonia had higher risk of DVT(14). Research clarified that some pathogens, especially bacteria had surface proteins and exotoxins leading to damaging endothelial cells, activating coagulation pathway and forming micro-thrombosis and DVT(17).

Immobility was a major risk factor for DVT in neurological diseases $(4,18)$.An observational research analyzed 542 stroke patients with DVT and found that DVT occurred in 73\% of patients with weaker muscle strength while only $11 \%$ of patients with stronger were diagnosed with DVT(19). Our study showed that there was no difference in muscle strength between DVT-group and non-DVT group. The difference could be partly explained by the different samples and subtypes of the patients. In our study, ischemic stroke accounted for $77.2 \%$,while others were hemorrhagic stroke patients. Some researches on different subtypes of stroke clarified that patients with hemorrhagic stroke had significantly higher risk of DVT due to lower rate of antithrombotic management and more severe neurological disability in patients with hemorrhagic stroke compared with patients with ischemic stroke(20). 
Although muscle strength was not linked with DVT in our study, muscle tone was identified as a negative relevant factor with the incidence of DVT and patients with increased muscle tone were less likely to developing DVT. Among patients with stroke, increased muscle tone and muscle spasms of lower extremities usually develop gradually within several months(21), which theoretically resulted in emptying of veins in lower extremities by enhancing the capability of the calf muscle pump. Previous studies observed some vascular changes with a generalized atrophy of the arteries and decreased blood flow in the paralyzed lower extremities, which could adjust the lower oxygen supply to match the decreased activity of the paralyzed muscles(22). With blood stasis reduced in extremities, the risk of DVT was decreased. Moreover, clinical observations suggested that increased muscle tone was a protective factor against DVT in neurological disorders $(19,23)$. Increased muscle tone in stroke patients at the initial stage indicated the gradual emergence of active exercise, which could lead to increase the cerebral blood flow in the injured site and promote the recovery of motor function and intelligence, resulting in blood flow velocity of hemiplegic extremities increased and the occurrence of DVT decreased $(24,25)$.

Interestingly, we compared the laboratory variables in different times and found that the level of D-dimer was significantly higher when DVT detected than that on admission, which suggested that dynamically testing D-dimer could be a predictive method for DVT. D-dimer as a sensitive marker for thrombus formation ,was an indicator for predicting DVT in different disorders $(12,15,26)$. In the current studies, Ddimer demonstrated a sensitivity of $85 \%-95 \%$ and a specificity of $25 \%-50 \%$ for DVT $(27,28)$. Baseline levels of D-dimer varied in different age due to variability in the inflammatory and immune response dependent on age(29)and the elders were more likely to suffering from stroke, which could explain why the specificity of a standard D-dimer cut-off at $500 \mathrm{ug} / \mathrm{L}$ for DVT prediction in elderly patients with stroke was comparatively low. A systemic review indicated that utility of an age-adjusted D-dimer cut-off (patient's age*10)ug/L) for elderly patients for ruling out DVT was recommended(30). The average levels of D-dimer when DVT detected were about 4-fold as the levels of that on admission in our study, which suggested that the gradually increased level of d-dimer was associated with DVT.

The strength of this study is that it concludes that pulmonary infection as a risk factor and increased muscle tone as a protector factor for DVT, which enables physicians to take early managements to reduce the incidence of DVT such as paying more attention to the patients with pulmonary infection and taking more effective therapies to improve muscle tone of patients. In addition, the significant change in level of D-dimer could be a warning for DVT occurrence so that dynamically monitoring the level of Ddimer is of importance in patients after stroke.

There were some limitations. First, due to relatively small samples, we didn't divide the cohort into several subtypes groups such as ischemic stroke, hemorrhagic stroke and traumatic brain injury. Further study with larger samples and more subtypes needs to be explored. Second, because it was a retrospective research, the time when DVT detected by CDUS might be delayed compared to the actual time when DVT developed. Further prospective research should be performed to validate our conclusions. Moreover, bias couldn't be avoided. Caution should be needed while our findings is interpreted in other multiple-center cohort studies. Third, although venography was the gold standard for diagnosing DVT, serial 
compression ultrasonography as the reference test was applied in our study for detecting DVT owing to its non-invasiveness. It might be not as perfectly accurate as venography.

\section{Conclusion}

Pulmonary infection as a risk factor and increased muscle tone as a protector factor for DVT were identified in patients after acute stroke and the significant change in level of D-dimer could be a warning for DVT occurrence.

\section{List Of Abbreviations}

DVT=deep vein thrombosis, LOS=length of stay, NIHSS =National Institutes of Health Stroke Scale, GCS=Glasgow Coma Scale, MRS=Modified Rankin Scale, RDW=red blood cell volume distribution width, $\mathrm{LDL}=$ low density lipoprotein, $\mathrm{Fbg}=$ fibrinogen, $C D U S=$ color doppler ultrasonography, IQR= interquartile range, $\mathrm{OR}=$ Odds Ratio, $\mathrm{BMI}=$ body mass index

\section{Declarations}

\section{Acknowledgments}

No

\section{Funding}

This manuscript was supported by Health and Family Planning Commission of Hunan Province (CN) (NO.20180468).

\section{Ethics approval and consent to participate}

Ethics approval was provided by the Medical Ethics Committee of Changsha central Hospital. Due to the nature of retrospective study, informed consent was waived.

\section{Availability of data and materials}

Datasets used and/or analyzed in the present study were availed by the corresponding author on reasonable request.

\section{Author contributions}


The manuscript writing and patient's data recording were done by Yucai Huang and Ning Ding. Kun Song, Wen Peng and Yang Zhou assisted in information collection. Changluo Li and Ning Ding analyzed and interpreted the patients' general indices. The final manuscript was read and ratified by all authors.

\section{Disclosure Statement}

There are no real or apparent conflicts of interest to disclose.

\section{Consent for publication}

Not applicable

\section{References}

1. Powers WJ, Rabinstein AA, Ackerson T, et al. 2018 Guidelines for the Early Management of Patients With Acute Ischemic Stroke: A Guideline for Healthcare Professionals From the American Heart Association/American Stroke Association. Stroke. 2018;49(3):e46-e110.

2. Wu DM, Zheng ZH, Wang S, et al. Association between plasma macrophage migration inhibitor factor and deep vein thrombosis in patients with spinal cord injuries. Aging (Albany NY). 2019;11(8):2447-56.

3. Balogun IO, Roberts LN, Patel R, et al. Clinical and laboratory predictors of deep vein thrombosis after acute stroke. Thromb Res. 2016;142:33-9.

4. Liu LP, Zheng HG, Wang DZ, et al. Risk assessment of deep-vein thrombosis after acute stroke: a prospective study using clinical factors. CNS Neurosci Ther. 2014;20(5):403-10.

5. Naccarato M, Chiodo Grandi F, Dennis M,et al. Physical methods for preventing deep vein thrombosis in stroke. Cochrane Database Syst Rev. 2010(8):Cd001922.

6. Dennis M, Sandercock P, Reid J, et al. Can clinical features distinguish between immobile patients with stroke at high and low risk of deep vein thrombosis? Statistical modelling based on the CLOTS trials cohorts. J Neurol Neurosurg Psychiatry. 2011;82(10):1067-73.

7. De Silva DA, Pey HB, Wong MC,et al. Deep vein thrombosis following ischemic stroke among Asians. Cerebrovasc Dis. 2006;22(4):245-50.

8. Dizon MAM, De Leon JM. Effectiveness of Initiating Deep Vein Thrombosis Prophylaxis in Patients With Stroke: An Integrative Review. J Neurosci Nurs. 2018;50(5):308-12.

9. Yin D, Shao P, Liu Y. Elevated lipoprotein (a) levels predict deep vein thrombosis in acute ischemic stroke patients. Neuroreport. 2016;27(1):39-44.

10. Govender I, Mabuza HL, Ogunbanjo GA. The characteristics of HIV and AIDS patients with deep vein thrombosis at Dr. George Mukhari Academic Hospital. Afr J Prim Health Care Fam Med. 2015;7(1). 
11. Koyuncu E, Nakipoglu Yuzer GF,et al. Coexistence of Deep Vein Thrombosis, Heterotopic Ossification, and Complex Regional Pain Syndrome due to Hemorrhagic Stroke. J Stroke Cerebrovasc Dis. 2016;25(3):e38-40.

12. Fujiwara R, Numao N, Ishikawa $Y$, et al. Incidence and Predictors of Deep Vein Thrombosis in Patients with Elevated Serum D-Dimer Prior to Surgery for Urologic Malignancy. Urol Int. 2020;104(12):16-21.

13. Deshpande CG, Kogut S, Laforge R,et al. Impact of medication adherence on risk of ischemic stroke, major bleeding and deep vein thrombosis in atrial fibrillation patients using novel oral anticoagulants. Curr Med Res Opin. 2018;34(7):1285-92.

14. Martin E, Cevik C, Nugent K. The role of hypervirulent Staphylococcus aureus infections in the development of deep vein thrombosis. Thromb Res. 2012;130(3):302-8.

15. Demelo-Rodriguez P, Cervilla-Munoz E, Ordieres-Ortega L, et al. Incidence of asymptomatic deep vein thrombosis in patients with COVID-19 pneumonia and elevated D-dimer levels. Thromb Res. 2020;192:23-6.

16. Funayama M, Takata T. Psychiatric inpatients subjected to physical restraint have a higher risk of deep vein thrombosis and aspiration pneumonia. Gen Hosp Psychiatry. 2020;62:1-5.

17. Vardakas KZ, Matthaiou DK, Falagas ME. Comparison of community-acquired pneumonia due to methicillin-resistant and methicillin-susceptible Staphylococcus aureus producing the PantonValentine leukocidin. Int J Tuberc Lung Dis. 2009;13(12):1476-85.

18. Kelly J, Rudd A, Lewis RR,et al. Venous thromboembolism after acute ischemic stroke: a prospective study using magnetic resonance direct thrombus imaging. Stroke. 2004;35(10):2320-5.

19. Gaber TA. Significant reduction of the risk of venous thromboembolism in all long-term immobile patients a few months after the onset of immobility. Med Hypotheses. 2005;64(6):1173-6.

20. Ji R, Li G, Zhang R,et al. Higher risk of deep vein thrombosis after hemorrhagic stroke than after acute ischemic stroke. J Vasc Nurs. 2019;37(1):18-27.

21. Dennis M, Mordi N, Graham C,et al. The timing, extent, progression and regression of deep vein thrombosis in immobile stroke patients: observational data from the CLOTS multicenter randomized trials. J Thromb Haemost. 2011;9(11):2193-200.

22. Stavres J, Singer TJ, Brochetti A,et al. The Feasibility of Blood Flow Restriction Exercise in Patients With Incomplete Spinal Cord Injury. PM R. 2018;10(12):1368-79.

23. Green D, Hartwig D, Chen D, et al. Spinal Cord Injury Risk Assessment for Thromboembolism (SPIRATE Study). Am J Phys Med Rehabil. 2003;82(12):950-6.

24. Thijssen DH, De Groot PC, van den Bogerd $A$, et al. Time course of arterial remodelling in diameter and wall thickness above and below the lesion after a spinal cord injury. Eur $\mathrm{J}$ Appl Physiol. 2012;112(12):4103-9.

25. Douce D, McClure LA, Lutsey P, et al. Outpatient Treatment of Deep Vein Thrombosis in the United States: The Reasons for Geographic and Racial Differences in Stroke Study. J Hosp Med. 2017;12(10):826-30. 
26. Chi G, Goldhaber SZ, Hull RD, et al. Thrombus Burden of Deep Vein Thrombosis and Its Association with Thromboprophylaxis and D-Dimer Measurement: Insights from the APEX Trial. Thromb Haemost. 2017;117(12):2389-95.

27. Nybo M, Hvas AM. Age-adjusted D-dimer cut-off in the diagnostic strategy for deep vein thrombosis: a systematic review. Scand J Clin Lab Invest. 2017;77(8):568-73.

28. Schouten HJ, Koek HL, Oudega R, et al. Validation of two age dependent D-dimer cut-off values for exclusion of deep vein thrombosis in suspected elderly patients in primary care: retrospective, cross sectional, diagnostic analysis. Bmj. 2012;344(jun06 1):e2985-e.

29. Pawelec G, Goldeck D, Derhovanessian E. Inflammation, ageing and chronic disease. Curr Opin Immunol. 2014;29:23-8.

30. Prochaska JH, Frank B, Nagler M, et al. Age-related diagnostic value of D-dimer testing and the role of inflammation in patients with suspected deep vein thrombosis. Sci Rep. 2017;7(1):4591.

\section{Figures}

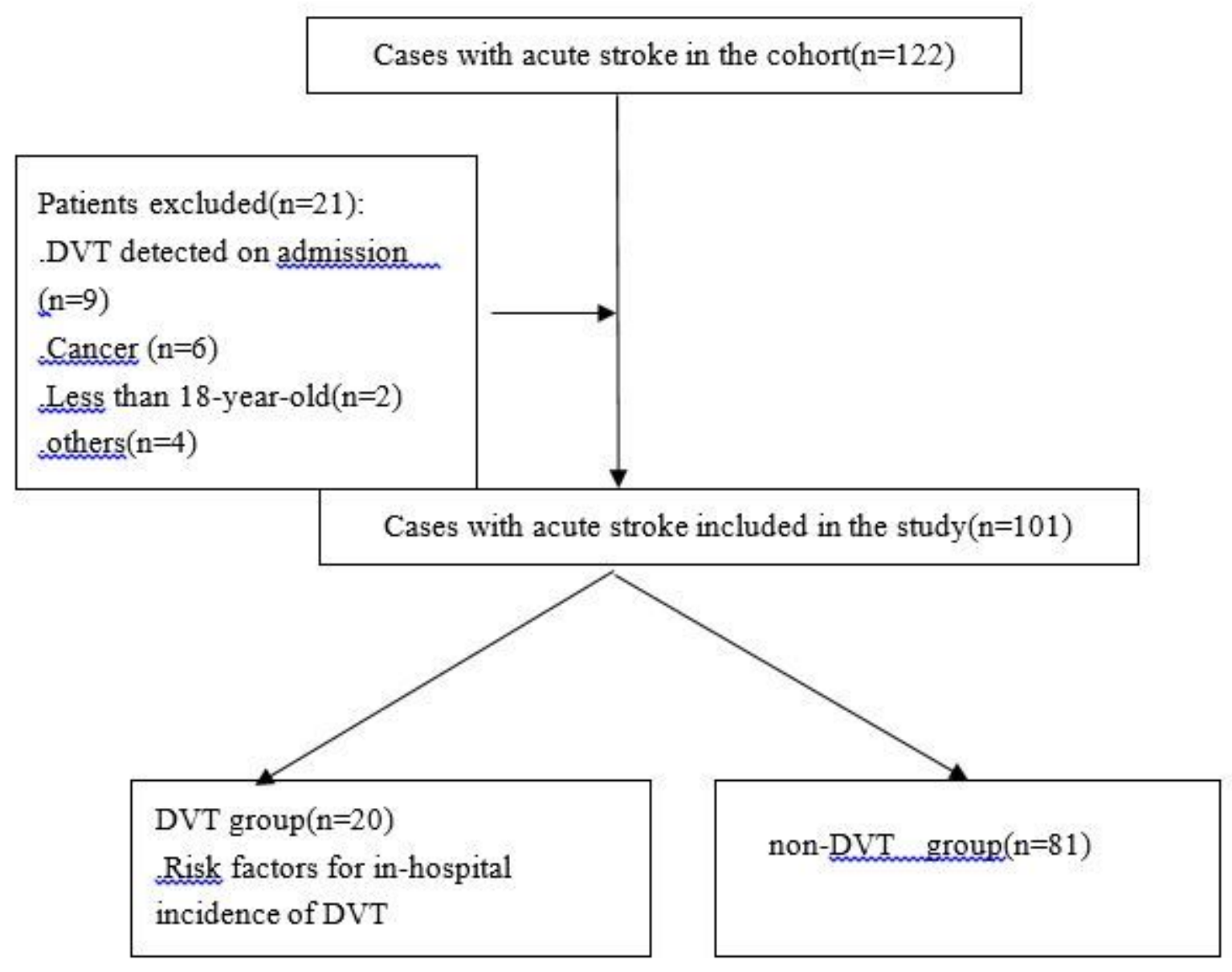

Figure 1 
Flow chart for patients enrollment and study design.

\begin{tabular}{|c|c|c|}
\hline 40 & & $67.8 \%$ \\
\hline 35 & & \\
\hline 30 & & \\
\hline 25 & & \\
\hline 20 & & \\
\hline 15 & & \\
\hline 10 & & \\
\hline 5 & $10 \%$ & \\
\hline 0 & DVT group & non-DVP group \\
\hline muscle tone increased & 2 & 38 \\
\hline
\end{tabular}

Figure 2

Comparison muscle tone between DVT and non-DVT groups (10\%vs67.8\%,P=0.002)

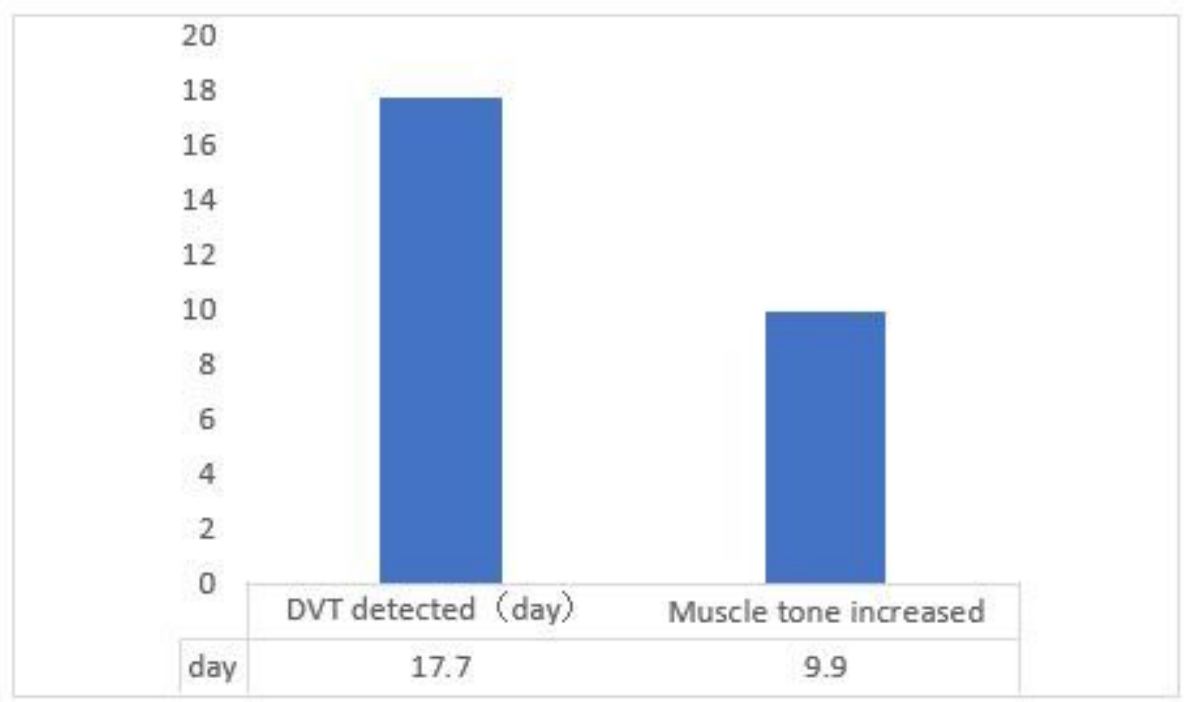

Figure 3

Comparison the time between when DVT detected and when muscle tone increased $\llbracket 17.7 v s 9.9, P<0.001 \rrbracket$ 